\title{
Couplage électro-thermomécanique d'un assemblage BGA soumis à des cycles combinées et alternées de puissance et thermique
}

\author{
Electro thermomechanical coupling of a BGA assembly subjected to \\ combined and alternating power and thermal cycles
}

\author{
Ghenam Sinda $^{1,2}$, El Hami Abdelkhalak ${ }^{1}$, Gafsi Wajih², Akrout Ali $^{2}$, Haddar Mohamed $^{2}$ \\ ${ }^{1}$ LMN, INSA - Rouen, Rouen, France, sinda.ghenam@enis.tn, abdelkhalak.elhami@insa-rouen.fr \\ ${ }^{2}$ LA2MP, ENIS, Sfax, Tunisie, wajih.gafsi@enis.tn, ali.akrout2015@gmail.com, mohamed.haddar@enis.rnu.tn
}

\begin{abstract}
RÉSUMÉ. La légèreté des dispositifs électroniques et la progression de la densité de puissance amènent les concepteurs à améliorer constamment les performances des systèmes électroniques. Parmi les charges fréquentes que subit cet équipement figurent les cycles d'alimentation électriques et les cycles thermiques. La combinaison de ces cycles conduit souvent à la défaillance du dispositif électronique et par conséquent à la défaillance de l'ensemble du système. Parmi les composants les plus sollicités dans ces équipements, on trouve les joints de soudure dont le matériau est le SAC305. Dans cette étude, le but est d'étudier l'évolution du gradient thermique dans le cas du Power Cycling et du Thermal Cycling, soit alternés, donc il s'agit des effets Joule et Peltier seuls, soit combinés, donc il s'agit de la combinaison des deux effets cités précédemment plus le flux de chaleur généré mors de la soumission de l'équipement dans une chambre thermique virtuelle et son impact sur la déformation thermique des joints de brasure. La méthode des éléments finis (FEM) est utilisée pour simuler les champs électriques, thermiques et mécaniques couplées dans l'assemblage et pour évaluer la réponse des joints de soudure exposés au processus mentionné. Les contraintes et les déformations sont évalués.
\end{abstract}

ABSTRACT. Lightweight electronic devices and increasing power density are driving designers to constantly improve the performance of electronic systems. Among the frequent loads experienced by this equipment are electrical power cycles and thermal cycles. The combination of these cycles often leads to the failure of the electronic device and consequently to the failure of the entire system. Among the most stressed components in this equipment are the solder joints, whose material is SAC305. In this study, the aim is to study the evolution of the thermal gradient in the case of Power Cycling and Thermal Cycling, either alternated, so it is the Joule and Peltier effects alone, or combined, so it is the combination of the two effects mentioned above plus the heat flow generated during the submission of the equipment in a virtual thermal chamber and its impact on the thermal deformation of solder joints. The finite element method (FEM) is used to simulate the coupled electrical, thermal and mechanical fields in the assembly and to evaluate the response of the solder joints exposed to the mentioned process. Stresses and Strains are evaluated.

MOTS-CLÉS. Cycle d'alimentation, cycle thermique, joints de soudure SAC305, gradient thermique, effet joules, effet Peltier, déformation thermique, simulation électro-thermomécanique.

KEYWORDS. Power cycle, thermal cycle, SAC305 solder joints, thermal gradient, Joule effect, Peltier effect, thermal deformation, electro-thermomechanical simulation.

\section{Introduction}

Dans le domaine de l'électronique embarquée, l'impact de la bonne conception d'une structure est très important pour son fonctionnement, sa résistance et sa durée de vie. L'amélioration des techniques de conception des microstructures évolue de manière très remarquable. Les cartes électroniques sont des organes qui remplissent des fonctions stratégiques dans les systèmes embarqués. Elles doivent répondre à des exigences de fiabilité élevées durant leur cycle de vie. L'équipement électronique se constitue de nombreux composants avec des matériaux différents. La fatigue concerne tous les composants de ces équipements et surtout les interfaces de deux matériaux différents. Au cours du l'échauffement de l'équipement électronique, les matériaux se dilatent à des amplitudes différentes ce qui induit des contraintes et des déformations. Ces déformations peuvent devenir irréversibles et permanentes au cours des cycles thermiques. 
La légèreté des dispositifs électroniques et la progression de la densité de puissance amènent les concepteurs à améliorer constamment les performances des systèmes électroniques. Les systèmes à forte puissance de calcul utilisent souvent des réseaux de billes (BGA). Dès la phase de développement, les BGA nécessitent une conception et une optimisation solides pour garantir un contact fiable avec le PCB. Dans ce contexte, les résultats de la soudure peuvent être améliorés par des simulations. L'interconnexion des composants avec le circuit imprimé est réalisée entre les zones de report en cuivre sur la carte électronique et les terminaisons métalliques côté composant. Cette interconnexion est assurée par les joints de brasage. Les joints de brasage sont les organes les plus sollicités parmi les trois éléments principaux d'un assemblage électronique (composants, carte nue, joints brasés). De nombreuses causes de défaillances peuvent être recensées dans un équipement électronique : par exemple des défauts du composant ou de la carte assemblée. Cependant, la défaillance de l'assemblage se localise généralement dans le joint brasé. La tenue mécanique de ces joints dépend de nombreux paramètres dont principalement la conception de la carte assemblée (alliage sélectionné, géométrie de la brasure, conception de la carte imprimée, matériaux de base et critères de qualité de fabrication) et le profil de mission de l'équipement électronique. [ASS13], [DOM11].

La fatigue thermique des joints brasés est la cause majeure de la défaillance des équipements électroniques. Cette fatigue thermique est induite par les différents matériaux employés et possédant des propriétés hétérogènes. Ces équipements électroniques reposent en effet sur une structure complexe où des matériaux possédant des coefficients de dilatation thermique différents sont mis en contact. Sous des sollicitations thermiques dues à l'environnement extérieur ou à l'échauffement des composants par effet Joule, ou par l'effet Peltier généré entre deux matériaux différents, le différentiel de dilatation entre les différents constituants des composants et des assemblages fait apparaitre des déformations et des contraintes mécaniques. Ces déformations mécaniques engendrées peuvent être irréversibles à cause du comportement viscoplastique des matériaux utilisés et du phénomène du fluage. En outre, les principales causes de défaillance proviennent des contraintes de cisaillement générées par la dilatation différentielle au cours des cyclages thermiques. [ASS13].

L'article est structuré en cinq sections principales. La première section se concentre sur des élaborations mathématiques des équations pour les domaines électriques, thermiques et mécaniques. Pour la deuxième section, une brève explication des effets joules et Peltier est présentée. Dans la troisième section, une vue d'ensemble du système est illustrée, dans lequel nous décrivons la géométrie et les matériaux qui y sont attachés, en particulier le matériau SAC305 du joint de soudure en détaillant sa loi de comportement viscoplastique. Enfin, l'article se concentre sur la simulation numérique effectuée sur ANSYS Workbench afin de définir l'impact des cycles thermiques et de puissance superposés et alternés. Les cycles thermiques et électriques ont été simulés et les contraintes et déformations ont été évaluées et interprétées.

\section{Couplage électro-thermomécanique}

Les phénomènes physiques qui conditionnent le comportement électrique des dispositifs à semiconducteurs sont intimement liés à la température des composants. Et inversement, la température des composants est fortement liée à la dissipation de puissance. Il existe donc un couplage entre le comportement électrique des composants de puissance et l'impact thermique de l'ensemble de la structure. Ainsi, lors de la conception de l'ingénierie électrique, notamment des composants et systèmes d'électronique de puissance, l'évolution thermique au cours des cycles de fonctionnement doit être prise en compte afin d'augmenter la fiabilité des systèmes de puissance. Ce phénomène correspond à la création de chaleur à partir d'un flux de courant dans un corps : c'est l'effet joules. Quand il s'agit de création d'une chaleur entre deux matériaux différents suite au passage d'un courant électrique : on parle de l'effet Peltier. Les forts courants qui vont traverser ce composant lorsqu'il est en marche, vont provoquer un échauffement de la puce de puissance. Cette chaleur est 
évacuée par le boîtier qui l'échauffe à son tour. Les variations des matériaux utilisés dans la construction de ce composant, génèrent lors des variations de température, des contraintes thermomécaniques entre les différents matériaux qui le composent. Il est possible de faire un parallèle entre la chaleur et l'électricité car les lois fonctionnent de la même manière. C'est dans ce contexte qu'une analogie électrique-thermique a été faite. [MAK17]

\subsection{Domaine électrique}

Une tension électrique est appliquée sur la surface supérieure de la couche de cuivre. Cette tension va être conduite d'où la nécessité d'étudier la conduction électrique. Le paramètre principal est la conductivité électrique notée $\sigma$ et elle est l'inverse de la résistivité $\rho$. Elle caractérise la capacité d'un matériau à laisser les charges électriques se déplacer librement et donc à permettre le passage d'un courant électrique. En général, l'expression de la conductivité est la suivante :

$\sigma\left(\mathbf{S} \cdot \mathbf{m}^{-1}\right.$ ou $(\Omega \cdot \mathbf{m})^{-1}=\frac{\mathbf{G}}{\mathrm{S}} \cdot \mathbf{L}=\frac{\mathbf{L}}{\mathbf{R} \cdot \mathbf{S}}$

Avec : $\mathrm{G}$ : conductance, $\mathrm{S}$ : section, $\mathrm{L}:$ longueur.

Il existe deux types de conduction électrique :

$\checkmark$ La conduction électrique : le passage du courant à travers la pièce à chauffer.

$\checkmark$ La conduction indirecte : par l'échauffement d'une résistance.

Dans notre cas, nous allons étudier la conduction directe.

Lors de son fonctionnement, le BGA dissipe une puissance au niveau de la zone active de cette assemblage, qui est la puce en silicium principalement, par le phénomène auto-échauffement. Cette puissance est en fonction de la tension et le courant qui circule entre la puce et les billes de brasures en premier lieu and entre les billes et la couche de cuivre et FR4 en deuxième lieu. [AMA20]

\subsection{Domaine thermique}

La chaleur est générée dans les zones actives des puces semi-conductrices par la dissipation de puissance sous forme de pertes. Un transfert de chaleur qui devrait être appelé transfert thermique est un transit d'énergie sous une forme microscopique désordonnée. On dit de deux corps ayant la même température qu'ils sont en "équilibre thermique". Si leur température est différente, le corps le plus chaud cède de l'énergie au corps le plus froid : il y a transfert thermique, ou par la chaleur. Sous l'influence d'un gradient de température, la chaleur circule par conduction des hautes vers les basses températures. Lorsque deux systèmes sont à des températures différentes, le système le plus chaud cède de la chaleur au système le plus froid. Il y a échange ou transfert de chaleur entre ces deux systèmes. La quantité de chaleur transférée par unité de temps et par unité de surface de la surface isotherme est appelée densité de flux thermique $\phi$. [ZHA13]

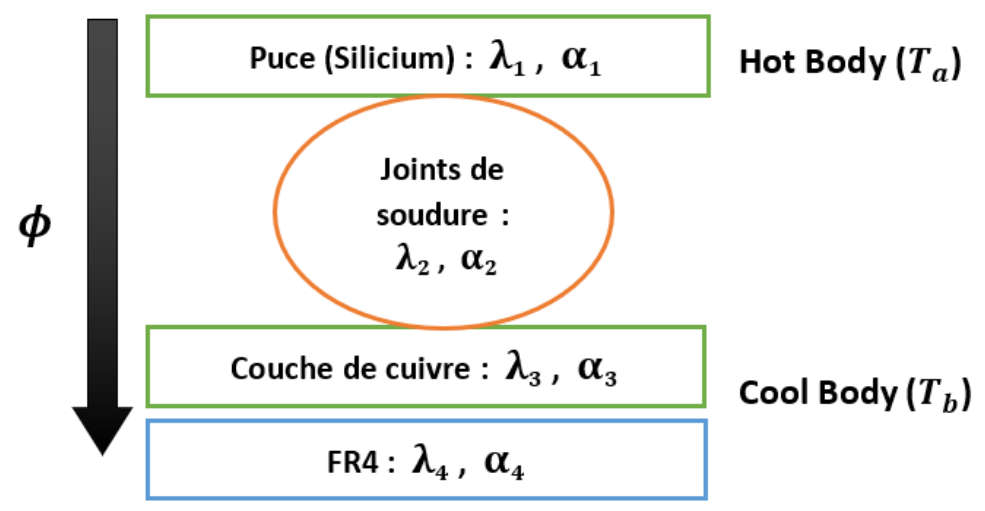

Figure 1. Figure explicative de la différence des coefficients d'expansion thermique 
Le transfert de chaleur se résument en trois phénomènes physiques suivants : La conduction qui se produit à l'intérieur des volumes des matériaux qui composent les structures, la convection qui est l'échange de chaleur avec le milieu environnant avec déplacement de fluide et la radiation qui intervient à haute température et notamment dans le monde de l'espace. Dans notre cas d'étude, on ne prend en considération que la conduction et la convection.

\section{Conduction thermique}

Si un gradient de température existe dans un matériau homogène isotrope, il y a un transfert d'énergie thermique sous forme de conduction thermique. [ZHA13]

Ce phénomène, en régime permanant suit l'équation suivante :

$\mathbf{Q} \cdot \mathbf{c}_{\mathbf{p}} \cdot \frac{\partial \mathbf{T}}{\partial \mathbf{t}}=\lambda \frac{\partial^{2} \mathbf{T}}{\partial \mathbf{x}^{2}}+\mathbf{Q}$

Q: masse volumique ; $\mathrm{c}_{\mathrm{p}}$ :capacité calorifique ; $\mathrm{T}$ : température en $\mathrm{K} ; \mathrm{Q}$ : puissance volumique générée dans le corps ; $\lambda$ : conductivité thermique

La loi de Fourrier postule que dans un corps homogène ou il y a dissipation de puissance sous forme de chaleur, le flux de chaleur est proportionnel au gradient de température :

$\overrightarrow{\mathbf{q}}=\lambda \overrightarrow{\operatorname{grad}}(\mathbf{T})$

\section{Convection}

Quand un solide est en présence d'un fluide de température différente, il se produit un transfert d'énergie par le mouvement des molécules dans le fluide, ce phénomène est appelé convection. [ZHA13]. Le mécanisme de transfert est régi par la loi de Newton :

$\vec{\varphi} \cdot \overrightarrow{\mathbf{n}}=\mathbf{h}\left(\mathrm{T}_{\mathrm{f}}-\mathrm{T}_{\mathrm{s}}\right)$

Avec $: \mathrm{h}$ : le coefficient de transfert de chaleur ; $\mathrm{T}_{\mathrm{s}}$ : la température du fluide au contact de la surface ; $\mathrm{T}_{\mathrm{f}}$ : la température de la surface du modèle.

\subsection{Analogie Electrique-thermique :}

En considérant l'analogie électrique - thermique, on obtient les relations suivantes :

$\mathrm{V}:$ potentiel électrique $\rightarrow \mathrm{T}:$ Temperature

$\mathrm{R}$ : résistance électrique $\rightarrow \mathrm{R}_{\mathrm{Th}}$ : résistance thermique

$\sigma:$ conductivité électrique $\rightarrow \boldsymbol{\lambda}$ : conductivité thermique

I : Intensité électrique $\rightarrow \phi:$ flux de chaleur

\subsection{Domaine mécanique :}

Le principal paramètre responsable des contraintes mécaniques dans les assemblages de puissance est la différence des coefficients de dilatation thermiques des matériaux assemblés qui entraîne des déformations différentes entre ces derniers en créant des contraintes en particulier aux interfaces. D’une manière générale, les contraintes mécaniques provoquées par les sollicitations thermiques peuvent être des contraintes de traction-compression $\left(\boldsymbol{\sigma}_{\mathrm{x}}, \boldsymbol{\sigma}_{\mathrm{y}}, \boldsymbol{\sigma}_{\mathrm{z}}\right)$ ou de cisaillement $\left(\boldsymbol{\tau}_{\mathrm{xy}}, \boldsymbol{\tau}_{\mathrm{xz}}, \boldsymbol{\tau}_{\mathrm{yx}}, \boldsymbol{\tau}_{\mathrm{yz}}, \boldsymbol{\tau}_{\mathrm{xx}}, \boldsymbol{\tau}_{\mathrm{zy}}\right)$. Pour estimer la valeur moyenne des contraintes que peut subir un matériau en un point, une grandeur a été définie appelée contrainte de Von Mises dont l'expression 
est donnée par l'équation suivante. Cette grandeur est couramment utilisée dans les études numériques pour l'estimation de la résistance des matériaux dans les assemblages de puissance: [BOU08]

$S=\sqrt{\frac{1}{2}\left[\left(\sigma_{x}-\sigma_{y}\right)^{2}+\left(\sigma_{y}-\sigma_{z}\right)^{2}+\left(\sigma_{z}-\sigma_{x}\right)^{2}+6\left(\tau_{x y}^{2}+\tau_{y z}^{2}+\tau_{z x}^{2}\right)\right]}$

\section{Effet Joules et effet Peltier}

Le passage du courant électrique dans les conducteurs s'accompagne de phénomènes thermiques. De façon plus générale, on rencontre toujours simultanément, dans les conducteurs ou aux jonctions des conducteurs, des phénomènes thermiques et des phénomènes électriques. Certains d'entre eux ne dépendent pas du sens du passage du courant (il y a toujours dégagement de chaleur par effet Joule, jamais absorption) ; d'autres, au contraire, en dépendent. Les phénomènes dits thermoélectriques sont de ceux -ci. Ils présentent en outre un caractère de réciprocité : à l'effet Peltier, qui relie l'échange de chaleur provoqué à une jonction de deux conducteurs au passage du courant, correspond l'effet Seebeck qui détermine la naissance d'un courant électrique dans un couple de conducteurs dont les températures sont différentes. [BON97] cet effet est expliqué et illustré dans la figure ci-dessous :

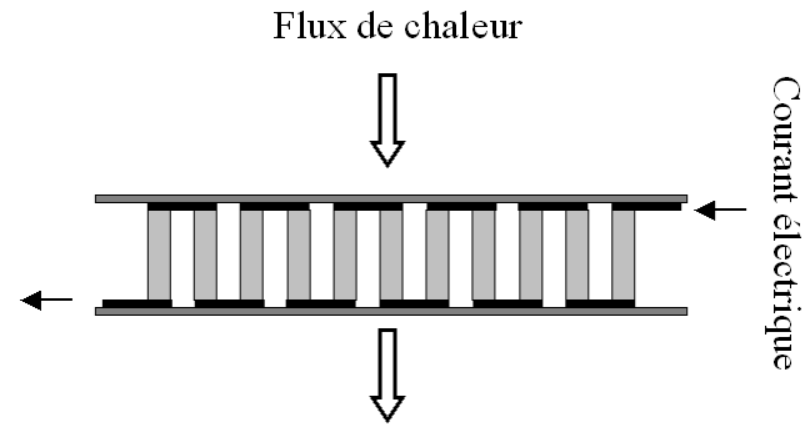

Figure 2. Illustration de l'effet Peltier

Quant à l'effet joules, il s'agit d'un courant provoque un échauffement de la matière. La puissance électrique transformée en puissance thermique est proportionnelle au carré du courant. Le facteur de proportionnalité $\mathrm{R}$ s'appelle la résistance.

Il est primordial de distinguer entre ces deux effets. Pour l'effet joules, il s'agit d'un dégagement de chaleur au sein d'un même matériau suite à la circulation du courant. Quant à l'effet Peltier, il est présent lorsqu'il s'agit de deux matériaux différents avec deux coefficients de dilation thermique (CTE) différents et qui sont en contact. Cet effet est important dans notre étude car il génère, en coopération avec l'effet joules, un gradient de température, susceptible de générer après une déformation thermique supplémentaire lorsqu'il s'agit de l'échauffement de l'air environnent de la carte électronique ou dans notre cas le BGA assembly dans une chambre thermique. Cette étude de gradient thermique dans le cas des APC (Accelerated Power Cycling) et ATC (Accelerated Thermal Cycling) soit alternés, donc il s'agit des effets joules et Peltier seules, ou combinées, donc il s'agit de la combinaison des deux effets mentionnés avant plus le gradient de température généré lors de ATC. 


\section{Description du système étudié}

Dans cet article, nous traiterons un composant électronique appelé BGA : Ball Grid Array soumis à différentes charges de cyclage qui seront simulées grâce à la méthode numérique des éléments finis Multiphysic. Les composants de type BGA (Ball Grid Array) sont équipés d'une matrice de billes sous le composant pour l'interconnexion avec la carte. Ainsi, lors de l'assemblage la composition du joint brasé dépend des billes, du composant et du métal d'apport. L'avantage principal de cette technique est d'offrir une plus grande densité d'interconnexion que les autres types de boîtiers. Les dimensions de la matrice de billes sont variables, de 5 à $50 \mathrm{~mm}$ de côté avec un écartement entre chaque bille compris entre 0,4 et 1,27mm. [ASS13] L'assemblage du composant BGA est composé d'un circuit imprimé (PCB) composé de deux couches de base : le FR4 et le cuivre. Le composant est monté avec une grille de $36(6 \times 6)$ billes de soudure.

Kariya et al. [KAR04] ont recommandé le SAC305 pour la bosse de soudure du flip-chip parce qu'ils ont supposé que la résistance et la ductilité à la fatigue du SAC305 sont mieux équilibrées par rapport au SAC405. En outre, le SAC305 est moins coûteux en raison de sa faible teneur en Ag par rapport au SAC405. De plus, l'association japonaise de développement de l'industrie électronique (JEIDA) a recommandé le SAC305. La soudure sans plomb Sn96 (SAC305) est un fil de soudure sans plomb de qualité électronique. Elle utilise la composition prédominante de l'alliage sans plomb. Cette soudure est une excellente alternative aux soudures au plomb. [DEP20] Les principales propriétés de ce type de soudure sont mentionnées ci-dessous :

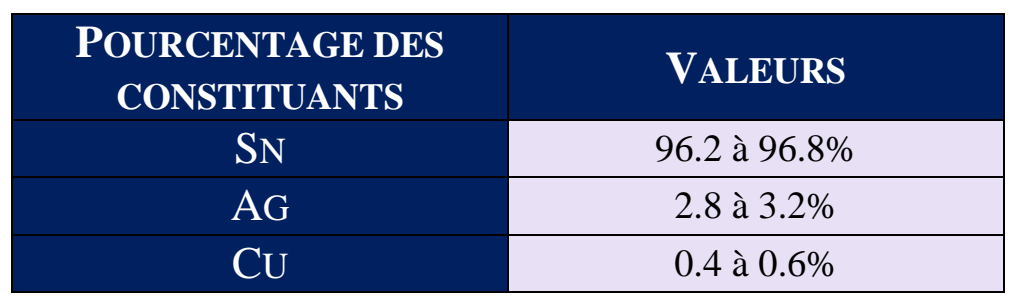

Tableau 1. Pourcentage des constituants de joints de soudure SAC305

Voici ci-dessous les paramètres géométriques du système étudié ainsi que les propriétés de matériaux des différents composants qui le composent en se basant sur des travaux antérieurs. [GHE22]

\begin{tabular}{|c|c|c|}
\hline & Dimensions $(\mathbf{m m})$ & Matériau \\
\hline Taille de la puce & $4^{\times} 4$ & Silicium \\
\hline Taille de PCB & $10^{\times} 10$ & Stratifié de FR4 et de cuivre \\
\hline Hauteur du paquet & 1.4 & SAC 305 \\
\hline Diamètre de la bille & 0.4 & --- \\
\hline Hauteur de bille & 0.8 & \\
\hline
\end{tabular}

Tableau 2. Dimensions et matériaux de l'assemblage BGA 


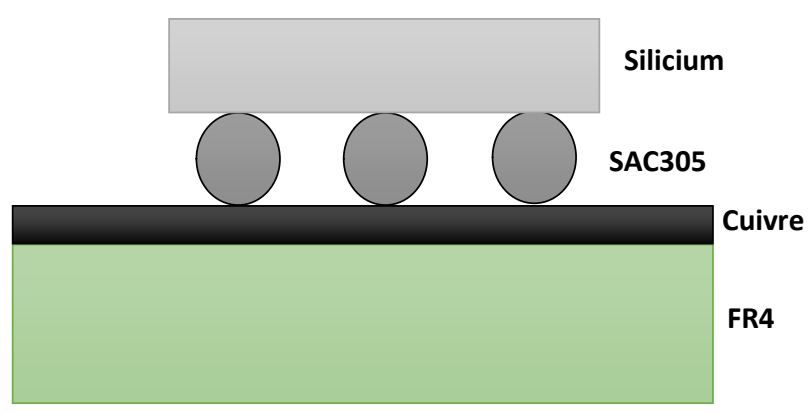

Figure 3. Géométrie du système BGA étudié

\begin{tabular}{|c|c|c|c|c|c|c|c|}
\hline & $\boldsymbol{\lambda}$ & $\boldsymbol{\alpha ( \mathbf { 1 0 - 6 } )}$ & $\boldsymbol{C p}$ & $\mathbf{E}(\mathbf{1 0 9})$ & $\boldsymbol{Q}$ & $\boldsymbol{v}$ & $\boldsymbol{\rho}$ \\
\hline FR4 & 0.3 & 18 & 1369 & 22 & 1900 & 0.28 & $8 \times 10^{11}$ \\
\hline Cuivre & 400 & 17 & 385 & 110 & 8960 & 0.35 & $1,7 \times 10^{-8}$ \\
\hline Silicium & 130 & 2.6 & 700 & 170 & 2330 & 0.28 & $4 \times 10^{3}$ \\
\hline SAC305 & 58 & 23.5 & 232 & 51 & 7380 & 0.4 & $1.23 \times 10^{-7}$ \\
\hline
\end{tabular}

Tableau 3. Propriètés des matériaux de l'assemblage BGA

\section{Lois de comportement des joints de soudure SAC305}

Parmi les différents modes de défaillance observés dans les assemblages électroniques, la fissuration par fatigue au niveau des joints de soudure semble être le plus critique. La détermination de la durée de vie d'un assemblage électronique en environnement sévère nécessite donc l'évaluation des contraintes et déformations mécaniques subies par les joints d'interconnexion lors des cycles de chargement. Dans le cadre de l'étude de la durabilité des équipements électroniques et donc de la tenue en fatigue thermomécanique et mécanique des joints de soudure, les lois de comportement les plus couramment utilisées sont basées sur la viscoplasticité. Plusieurs modèles ont été développés pour étudier le comportement viscoplastique des alliages de brasage. Nous citons principalement le modèle ANAND et le modèle BUSSO. BUSSO a développé un modèle viscoplastique pour l'alliage $\mathrm{Sn}-40 \mathrm{~Pb}$. Ce modèle décrit le comportement monotone et cyclique de l'alliage à différentes températures en tenant compte de l'écrouissage cinématique et du phénomène d'accommodation au cours des cycles. Quant au modèle d'Anand, il a été développé pour décrire la déformation des métaux à haute température (lorsque les phénomènes visqueux sont activés) en incluant la dépendance de la vitesse de déformation. Les alliages de type SAC sont utilisés pour les hautes températures. [HAM19]

Pour l'analyse thermomécanique, en utilisant le modèle local, le matériau du joint de soudure $(\mathrm{SnAgCu})$ est supposé avoir un comportement viscoplastique. Le développement de la déformation plastique dans le matériau du joint de soudure dépend du taux de chargement. De nombreux auteurs ont étudié la réponse des joints de soudure sans plomb $(\mathrm{SnAgCu})$ et ont proposé des équations pour modéliser cette réponse. L'un des modèles développés est le modèle ANAND qui intègre la viscoplasticité et la plasticité dépendant du temps. WANG et al [WAN06] proposent un cadre unifié pour le comportement viscoplastique des matériaux $\mathrm{SnAgCu}$ qui sont appelés les équations ANAND. C'est la raison pour laquelle de nombreuses études ont utilisé le modèle d'Anand dans la description des alliages SAC. Ce modèle est disponible sous le logiciel de modélisation par éléments finis ANSYS La loi d'évolution de la déformation inélastique est décrite par l'équation : [VAN17]

$\dot{\varepsilon}_{p}=A \exp \left(\frac{-Q}{R T}\right)\left[\sinh \left(\xi \frac{\sigma}{s}\right)\right]^{\frac{1}{m}}$ 
Les paramètres du matériau $\mathrm{SnAgCu}$ dans le modèle d'Anand sont obtenus à partir des résultats expérimentaux et des littératures précédents.[9] Ces paramètres sont présentés dans le tableau suivant :

\begin{tabular}{|c|c|c|c|c|c|c|c|c|c|}
\hline $\begin{array}{c}\text { Paramètres } \\
\text { de SAC305 }\end{array}$ & $\mathbf{A}(\mathbf{s}-\mathbf{1})$ & $\mathbf{Q} / \mathbf{R}\left({ }^{\circ} \mathbf{K}\right)$ & $\xi$ & $\mathbf{m}$ & $\mathbf{s}^{\wedge}$ & $\mathbf{n}$ & $\mathbf{h 0}(\mathbf{M P a})$ & $\mathbf{a}$ & $\mathbf{s 0}(\mathbf{M P a})$ \\
\hline Valeurs & $5.87 .10^{4}$ & 7460 & 2 & 0.0942 & 58.3 & 0.015 & 9350 & 1.5 & 45.9 \\
\hline
\end{tabular}

Tableau 4. Paramètres d'Anand pour le SAC305

L'affectation des matériaux à chaque composant de l'assemblage se fait principalement sur ANSYS WORKBENCH. L'assemblage BGA est maillé avec des éléments finis tétraédriques et cubiques. Le maillage est raffiné au niveau des billes de soudure et des deux surfaces de contact.

\section{Simulation numérique multiphysique}

Comme le processus de test de cyclage électrothermique est très long et coûteux, la modélisation par éléments finis est largement acceptée dans l'analyse de la mécanique et de la fiabilité des matériaux et des structures comme un boîtier électronique, en particulier pendant la phase de conception. La méthode FE donne des indications précieuses sur les caractéristiques d'évolution des états internes dans les joints de soudure. Pour ce papier, deux cas ont été étudiés :

Le premier cas est 1'application des cycles de puissance allant de 0 à $5 \mathrm{~V}$ en alternance avec des cycles de températures allant de $-40^{\circ} \mathrm{C}$ à $85^{\circ} \mathrm{C}$ avec un 'dwell time' de $20 \mathrm{~min}$.

$>$ Le deuxième cas est 1'application des cycles de puissance allant de 0 à $5 \mathrm{~V}$ en parallèle avec des cycles de températures allant de $-40^{\circ} \mathrm{C}$ à $85^{\circ} \mathrm{C}$ avec un 'dwell time' de $20 \mathrm{~min}$.

De même pour les cycles de températures, ils sont au nombre de 6. Dans le but de bien décrire les chargements cycliques appliqués, deux courbes pour les deux cas ont été faites et sont présentés cijoint :

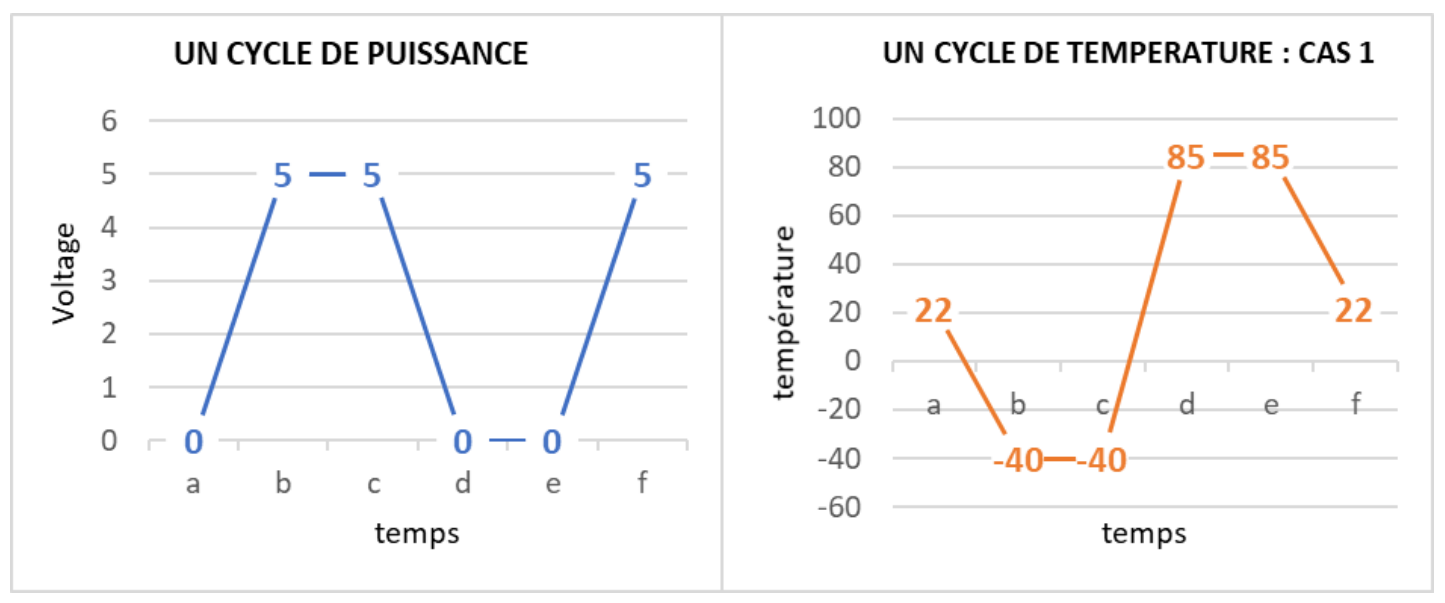

Figure 4. Cas 1 : Cycles alternées 


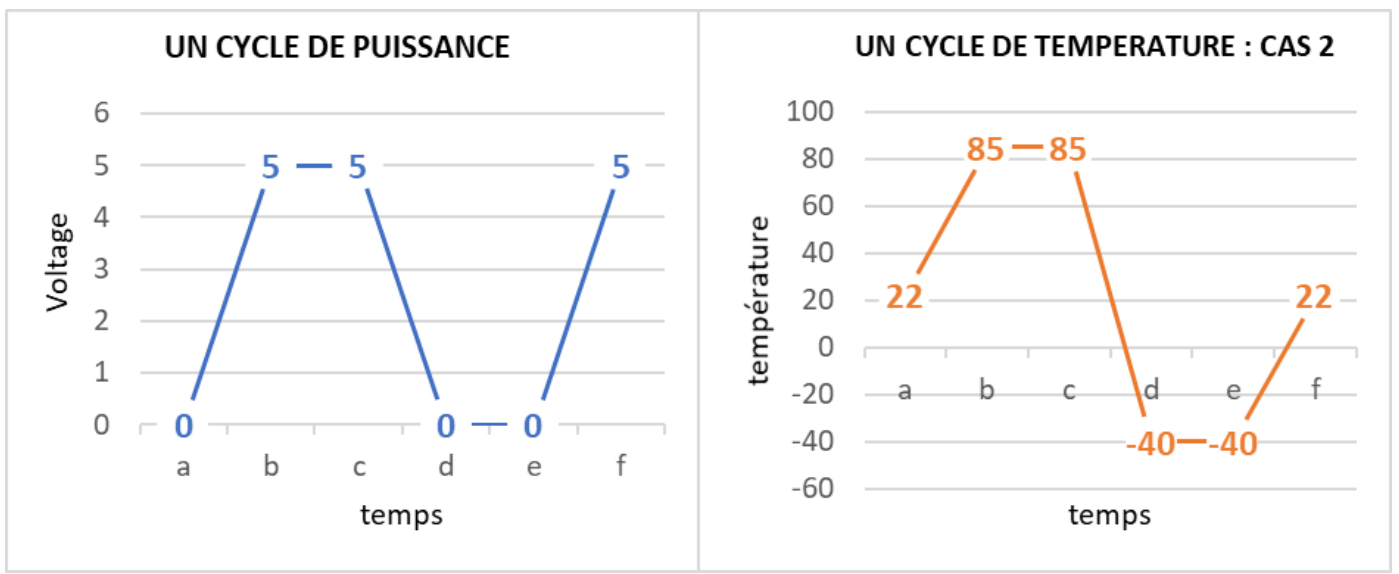

Figure 5. Cas 2 : Cycles superposées

Le but est d'étudier l'évolution du gradient thermique dans le premier cas et son impact majeur sur la déformation thermique majoritairement et sur le comportement mécanique en général. Pour le même objectif cette étude est menée dans le deuxième cas. Le résumé du but de ce papier figure dans le tableau ci-joint :

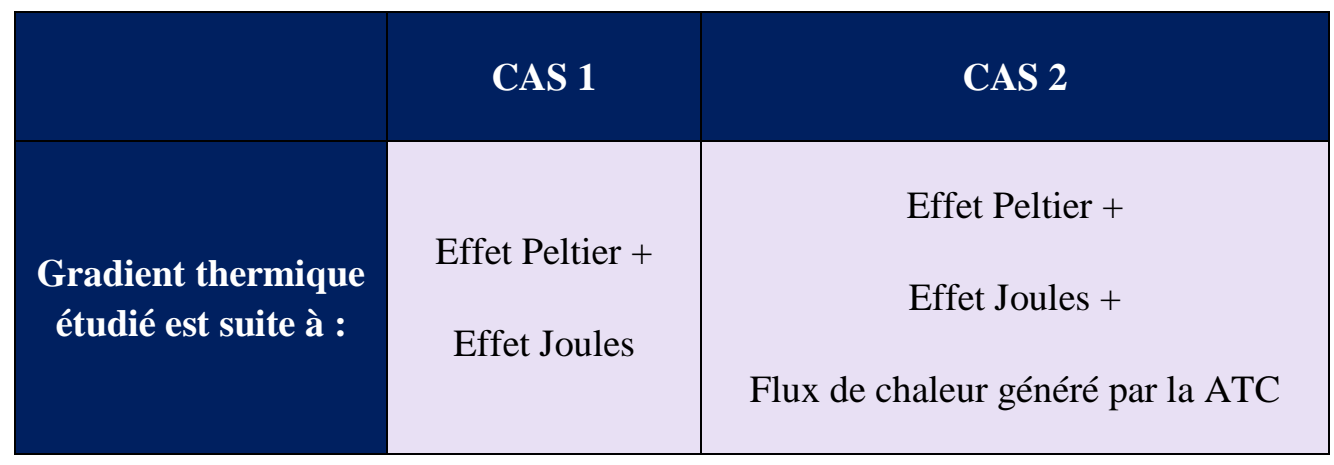

Tableau 5. Résumé de l'étude

\section{Résultats et discussion}

Suite à la résolution de cette simulation électro-thermomécanique, on a obtenu l'impact de cette charge cyclique sur le comportement mécanique de l'assemblage BGA tel que les contraintes équivalentes de Von Mises, les déformations élastiques et les déformations plastiques ainsi que l'énergie de déformation. En premier lieu, suite à la charge électrique on obtient la densité de courant de l'ordre de $38,962 \mathrm{~A} / \mathrm{m}^{2}$ et l'effet joules de l'ordre de $3,527 \times 10^{-6} \mathrm{~W} / \mathrm{m}^{3}$. Quant à l'application du module thermique transitoire on obtient la somme totale du gradient thermique :

$\checkmark$ Cas 1 : Grad $(\mathbf{T})=2.109 \times 10^{5} \mathrm{~W} / \mathrm{m}^{2}$

$\checkmark$ Cas $2: \operatorname{Grad}(\mathbf{T})=3.4 \times 10^{5} \mathrm{~W} / \mathrm{m}^{2}$

La distribution du flux thermique a la même allure dans les deux cas, comme illustré dans la figure ci-dessous : 


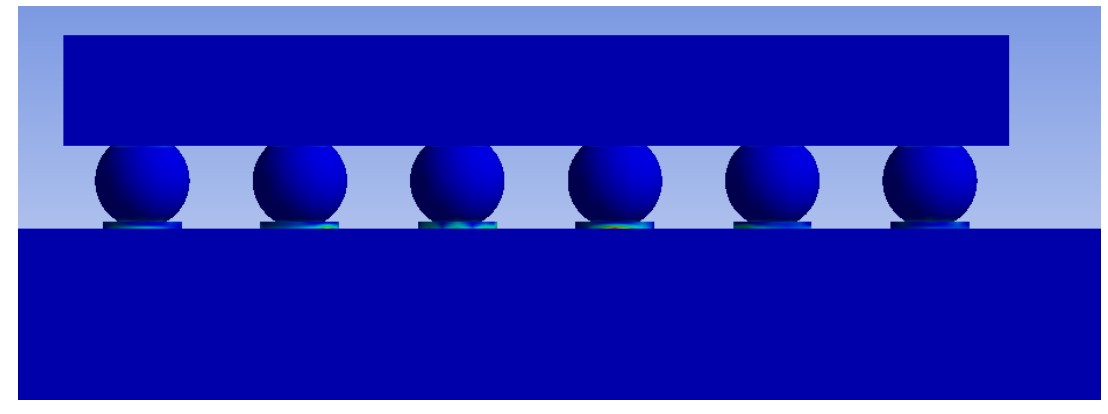

Figure 6. Distribution du flux thermique

Enfin, suite à cette charge electro-thermique, on obtient le comportement mécanique du BGA, présenté dans le tableau et les figures suivantes. Voici ci-joint le tableau résumant les résultats obtenus après simulation :

\begin{tabular}{|c|c|c|c|c|}
\hline Cycles & $\begin{array}{c}\text { Contrainte } \\
\text { équivalente } \\
(\mathbf{M P a})\end{array}$ & $\begin{array}{c}\text { Déformation totale } \\
\text { équivalente }\left(\mathbf{1 0}^{-4}\right)\end{array}$ & $\begin{array}{c}\text { Déformation } \\
\text { thermique }\left(\mathbf{1 0}^{-6}\right)\end{array}$ & $\begin{array}{c}\text { Energie de } \\
\text { déformation }\left(\mathbf{1 0} \mathbf{1 0}^{-9} \mathbf{J}\right)\end{array}$ \\
\hline Alternés & 16,68 & 6,78 & 4,69 & 1,5652 \\
\hline Superposés & 16,96 & 6,8942 & 93,66 & 1,6165 \\
\hline
\end{tabular}

Tableau 6. Résultats mécaniques obtenues de la simulation multiphysique des deux cas

Dans le premier cas d'étude, celui des cycles alternés, on a les valeurs de la contrainte équivalente de Von mises $\sigma_{\hat{e} q}$, de la déformation totale, la déformation thermique et l'énergie de déformation croient lorsque le gradient thermique que subit l'assemblage BGA croit. Ceci prouve que lorsqu'il s'agit des cycles combinés, les contraintes régis de l'effet joules sont additionnées avec celles régis de l'effet Peltier. D'où l'accumulation de ces deux types de contraintes, ce qui justifie l'augmentation des résultats mécaniques du deuxième cas par rapport au premier cas.

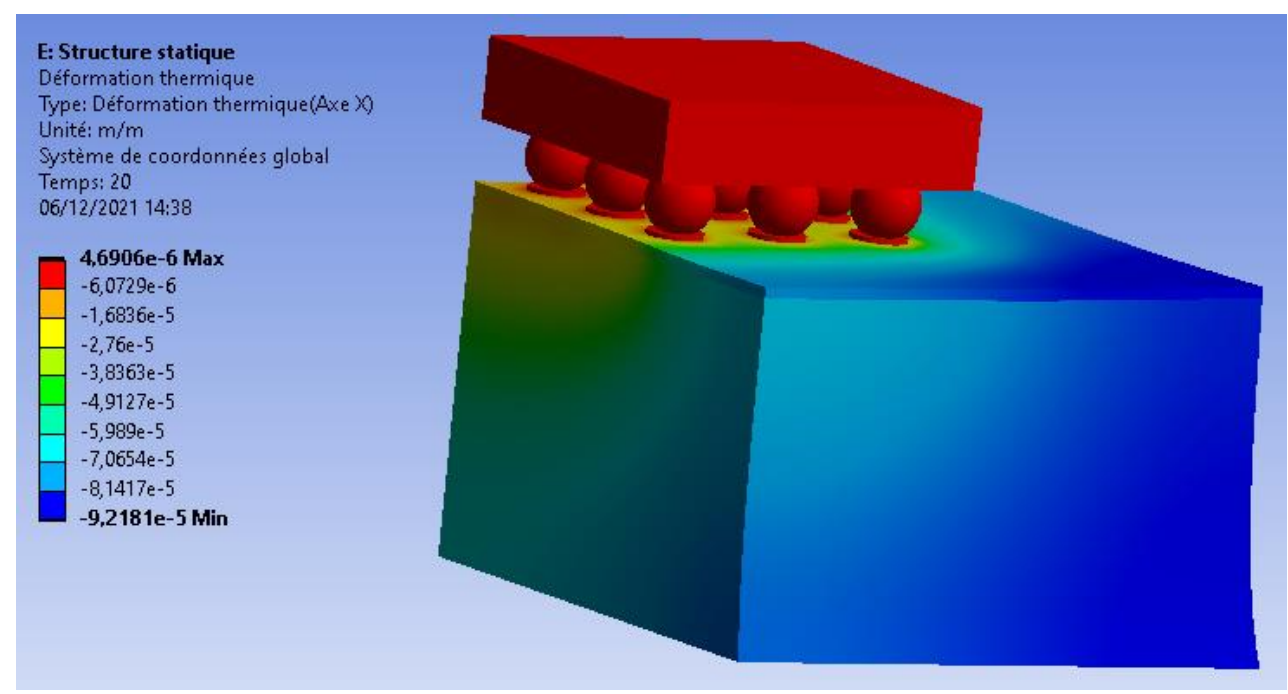

Figure 7. Distribution de la déformation thermique

La distribution de la contrainte et de la déformation est la même dans les deux cas. Elle est concentrée principalement dans les joints de brasures et particulièrement dans les bords de contact avec la puce en silicium et avec la couche de cuivre. Elle est décrite dans les figures ci-dessus.

Cette déduction confirme les travaux antécédents élaborés par les chercheurs tel que le travail de Joshua et al. [JOS20] Et l'étude faite par l'association iNEMI.[NEN10] 


\section{Conclusion}

Dans ce présent article, on a fait une modélisation électro-thermomécanique du composant BGA qui est l'un des composants essentiels dans un système mécatronique multiphysique. Cette étude a permis d'observer l'influence de la puissance dissipée par effet Peltier et l'effet joules combinées ou pas avec le flux thermique généré par la mise du composant dans une chambre thermique virtuelle sur le comportement mécanique du BGA tel que les contrainte équivalentes et les déformations élastiques et surtout thermiques en utilisant le modèle éléments finis résolus par le logiciel ANSYS WORKBENCH. Afin d'assurer une fabrication robuste des assemblages de BGA, donc celle des systèmes mécatroniques, il faut absolument faire une étude d'optimisation de la puissance dissipée donc par ailleurs, il faut étudier autres types de défaillances d'autres origines.

\section{Références}

[ASS13] ASSIF.S, EL HAMI A., and AGOUZOUL M., "Fiabilité et optimisation des structures mécaniques à paramètres incertains : application aux cartes électroniques", Thèse de doctorat, 2013.

[DOM11] DOMPIERRE B., CHARKALUK E., AUBIN V., "Fiabilité mécanique des assemblages électroniques utilisant des alliages du type SnAgCu,", Thèse de doctorat, Lille, France,2011.

[MAK17] MAKHLOUFI A., AOUES Y., EL HAMI A., RADI B., POUGNET P., and DELAUX D., "Study on the Thermomechanical Fatigue of Electronic Power Modules for Traction Applications in Electric and Hybrid Vehicles (IGBT)," in Reliability of High-Power Mechatronic Systems 1, Elsevier, 2017.

[HAM19] HAMDANI H., EL HAMI A., RADI B., "Métamodèles pour l'étude fiabiliste des systèmes mécatroniques", Thèse de doctorat, 2019.

[ZHA13] ZHANG Z., SCHNEIDER H., TOUNSI P., "Gestion thermique des composants d'électronique de puissance Utilisation du diamant CVD", Thèse de doctorat,2013.

[BOU08] BOUARROUDJ-BERKANI M., LEFEBVRE S., KHATIR Z., "Etude de la fatigue thermo mécanique de modules électroniques de puissance en ambiance de températures élevées pour des applications de traction de véhicules électriques et hybrides,", Thèse de doctorat, 2008.

[BON97] BONNISSEL M., TONDEUR D., "Adsorption de gaz modulée en température par des éléments thermoélectriques à effet Peltier.", Thèse de doctorat, 1997.

[KAR04] KARIYA Y., HOSOI T., TERASHIMA S., TANAKA M., OTSUKA M., "Effect of silver content on the shear fatigue properties of Sn-Ag-Cu flip-chip interconnects," Journal of Electronic Materials, vol. 33, no. 4, Apr. 2004.

[JOS20] DEPIVER J., MALLIK S., AMALU H., "Effective Solder for Improved Thermo-Mechanical Reliability of Solder Joints in a Ball Grid Array (BGA) Soldered on Printed Circuit Board (PCB)", Journal of Electronic Materials · November 2020.

[GHE22] GHENAM S., EL HAMI A., AKROUT A., GAFSI W., HADDAR M., "Electro-Thermomechanical Modelling of a BGA Assembly Subjected to a Damaging Displacement and to Random Vibrations,", papier de conference, Springer Nature, 2022, pp. 353-364.

[NEN10] HENSHALL G. et al. "iNEMI Lead-Free Alloy Alternatives Project Report: Thermal Fatigue Experiments and Alloy Test Requirements" 